\title{
Médiévales
}

Langues, Textes, Histoire

44 | printemps 2003

Le diable en procès

\section{Les who's who démonologiques de la Renaissance et leurs ancêtres médiévaux}

Demonological who's who of the 16th century and their medieval ancestors

Jean-Patrice Boudet

\section{(2) OpenEdition}

1 Journals

\section{Édition électronique}

URL : https://journals.openedition.org/medievales/1019

DOI : 10.4000/medievales. 1019

ISSN : $1777-5892$

Éditeur

Presses universitaires de Vincennes

Édition imprimée

Date de publication : 1 juin 2003

Pagination : $117-140$

ISBN : 2-84292-142-9

ISSN : 0751-2708

\section{Référence électronique}

Jean-Patrice Boudet, «Les who's who démonologiques de la Renaissance et leurs ancêtres médiévaux ", Médiévales [En ligne], 44 | printemps 2003, mis en ligne le 09 décembre 2005, consulté le 22 avril 2022. URL : http://journals.openedition.org/medievales/1019; DOI : https://doi.org/10.4000/ medievales.1019

Ce document a été généré automatiquement le 22 avril 2022.

Tous droits réservés 


\section{Les who's who démonologiques de la Renaissance et leurs ancêtres médiévaux}

Demonological who's who of the 16th century and their medieval ancestors

Jean-Patrice Boudet

1 Le Livre des esperitz, publié ci-dessous pour la première fois, est le plus ancien traité de magie démoniaque en français actuellement conservé. Les médiévistes peuvent certes faire la fine bouche en remarquant qu'il n'est pas si ancien que cela : ce texte, en effet, a été transcrit par une main anonyme sur un cahier de parchemin inséré dans le recueil factice du ms. Cambridge, Trinity College 0.8.29, aux fos $179-182 v^{\circ}$; or cette main, datée de la fin $d u \mathrm{Xv}^{\mathrm{e}}$ ou du début du Xvi ${ }^{\mathrm{e}}$ siècle par Elizabeth Butler ${ }^{1}$, et du $\mathrm{Xv}^{\mathrm{e}}$ siècle par Richard Kieckhefer ${ }^{2}$, ne me semble pas antérieure au milieu du xvI ${ }^{\mathrm{e}}$ siècle ${ }^{3}$. Mais cette date assez tardive n'enlève aucune valeur au document. Même s'il s'agit visiblement d'une traduction abrégée à l'usage d'un apprenti sorcier non latiniste ou mauvais latiniste, le Livre des esperitz est complet, ce qui nous permet d'apprécier sa structure d'ensemble : privilège rare et même unique, dans la mesure où, à ma connaissance, tous les autres catalogues de démons issus de l'Occident chrétien et antérieurs à 1600 nous sont parvenus sous une forme plus ou moins lacunaire.

2 Tel quel, ce texte est ce que je propose d'appeler un who's who démonologique : une liste de 46 démons, avec leur titre, leur apparence physique, leurs fonctions et le volume de leurs troupes, comptabilisé en légions. Après un bref paragraphe introductif qui reproduit le topos pseudépigraphique selon lequel le Livre des esperitz a été révélé à Salomon afin que la «malice » de ces créatures « ne regnast plus sur la terre crestienne $[s i c]$ », le document dresse leur nomenclature (deuxième paragraphe), puis donne une description relativement détaillée des trois super-démons que sont Lucifer, Gay/ Belzebuth et Satan. Viennent ensuite les quatre rois qui gouvernent les points cardinaux du ciel, Orient (Oriens), Poymon (Paymon), Amoymon (Amaymon) et Equi (alias Egyn, qui est oublié par la suite : il devrait donc y avoir 47 paragraphes et il y a 47 démons et non pas 46 ), puis, sans qu'un ordre hiérarchique soit suivi d'une manière 
continue, sept autres rois, huit princes, douze ducs, dix marquis, deux comtes et un " grant seigneur».

3 Cette sorte de petit bottin mondain démoniaque vise à être utilisée par un magicien, puisque le Livre des esperitz est suivi, aux fos $183-186 \mathrm{v}^{\circ}$, sur le même cahier de parchemin et de la main du même scribe, d'un Livre des conjuracions qui lui sert de mode d'emploi rituel, « par lequel l'on peult contraindre les esperitz [= les démons du Livre des esperitz] a faire obeÿr a humaine creature ». Or, premier indice de l'ancienneté du modèle sur lequel le Livre des esperitz a été traduit, ce Livre des conjuracions est une traductionadaptation d'un Liber consecrationum qui semble avoir été assez répandu au $\mathrm{xv}^{\mathrm{e}}$ siècle, puisque deux exemplaires différents de ce Liber se trouvent dans le ms. de Munich édité par R. Kieckhefer ${ }^{4}$, et qu'il en existe une autre version dans le ms. Oxford, Bodleian Library, Rawlinson D. 252, fos $81-87^{5}$.

Un autre témoin de la Renaissance

4 En 1577, le fameux démonologue brabançon Johannes Wier publie, sous le titre de Pseudomonarchia dcemonum, en annexe à la cinquième édition de son De prostigiis dæemonum, dont la première datait de 1563, un étonnant catalogue de 69 démons, suivi d'un bref modus operandi ${ }^{6}$. Dans un avis au lecteur ${ }^{7}$, il s'explique sur les motivations de cette étrange publication: soucieux de montrer au public jusqu'où peut aller la folie délirante des magiciens, il a extrait ce document des archives des vassaux de Satan, où l'on peut trouver des traités du même type, intitulés Officium spirituum (La fonction des esprits), Liber officiorum spirituum (Livre des fonctions des esprits), ou Liber dictus Empto[ris] (?) Salomonis, de principibus et regibus doemoniorum, qui cogi possunt divina virtute et humana (Livre, dit de Salomon le Simoniaque [?]', sur les princes et les rois des démons, qui peuvent être contraints par la force divine et humaine). L'ouvrage a fait forte impression et a été traduit en anglais par Reginald Scot, dans le livre XV de sa Discoverie of Witchcraft (1584) ${ }^{9}$.

D'emblée, une comparaison globale s'impose entre ces deux documents. Il en ressort qu'ils sont d'une nature analogue et ont un tronc commun mais que le premier n'est pas une traduction française du second : 35 démons du Livre des esperitz se retrouvent chez Wier (on les reconnaît parfois grâce à leurs attributs et fonctions, les noms étant fréquemment déformés et méconnaissables $:$ Gemer $=$ Buer, Machin $=$ Bathym, Bugan $=$ Haagenti, etc.), mais 15 démons du Livre des esperitz n'apparaissent pas dans la Pseudomonarchia dæemonum, et 31 démons de la Pseudomonarchia ne sont pas mentionnés dans le Livre des esperitz ${ }^{10}$. Nous avons donc affaire à deux versions distinctes d'un même type de texte, la version latine, bien que nettement plus longue, étant d'ailleurs la moins complète : le texte de la Pseudomonarchia semble amputé du début, puisque les deux premiers rois de Wier, Bael et Agares, sont de potestate ou sub potestate Orientis (comme l'est d'ailleurs Beal, dans le $\$ 7$ du Livre des esperitz), alors qu'il n'est pas question plus haut d'Oriens. Même remarque pour Paymon qui obedit magis Lucifer quam alii reges, alors qu'aucune notice particulière n'est consacrée à Lucifer. Il manque donc vraisemblablement au début de la Pseudomonarchia plusieurs paragraphes qui l'auraient rendu encore plus sulfureux sur Lucifer, Belzebuth, Satan, et les quatre démons des points cardinaux, dont Oriens. Par ailleurs, le modus operandi de la fin de la Pseudomonarchia est beaucoup plus rapide que le Liber consecrationum/Livre des conjuracions, et il présente des discordances anormales avec le catalogue de démons qui le précède ${ }^{11}$. Comme l'avait annoncé Wier dans son avis au lecteur, le texte de la Pseudomonarchia a donc été censuré en plusieurs endroits et rendu ainsi inutilisable pour le curiosulus qui aurait voulu en profiter pour jouer au magicien ${ }^{12}$. 
6 En revanche, le texte du démonologue est beaucoup plus détaillé et circonstancié que celui du Livre des esperitz sur l'origine des démons, leurs fonctions et leur apparence. On apprend ainsi qu'au moins une vingtaine d'entre eux sont des anges déchus clairement identifiés comme tels, qui appartenaient avant leur chute à l'un des neuf ordres d'anges du Pseudo-Denys ${ }^{13}$, et que cinq d'entre eux ont même le fol espoir de rejoindre l'ordre des Trônes, après avoir purgé une peine de mille ou douze cents ans ${ }^{14}$ ! Le texte reproduit par Wier signale également, d'une façon lapidaire, que chaque légion comprend 6666 démons - référence évidente à 666, le chiffre de la Bête de l'Apocalypse. Cela implique, étant donné que les démons de la Pseudomonarchia dirigent au total 2253 légions, que le nombre des démons susceptibles de sévir en ce bas monde se monte à 15018498 , chiffre bien inquiétant pour un chrétien du temps des Réformes... Et Jean Delumeau de remarquer : « au XVI ${ }^{e}$ siècle, on apporte les précisions numériques [sur le nombre des démons] dont on s'était auparavant prudemment abstenu $»^{15}$. Affirmation erronée si l'on se réfère non seulement à la démonologie byzantine ${ }^{16}$, mais aussi à la démonologie médio-latine occidentale : tout indique, en effet, que le Livre des esperitz et la Pseudomonarchia domonum sont les témoins tardifs d'une tradition textuelle bien antérieure.

Une tradition ancienne

7 Sans remonter à l'Antiquité et au Testament de Salomon ${ }^{17}$, ignoré par le Moyen Âge latin, énumérons les principaux indices de l'existence de cette tradition.

8 1. Dans la version longue du Liber introductorius de Michel Scot (†1236), dans le chapitre intitulé De notitia artis nigromancie pertinentis ad ymagines, l'astrologue de Frédéric II mentionne, entre autres opuscules sulfureux, un Liber perditionis anime et corporis ille, "qui traite dans l'ordre de toutes les fonctions des démons et de leurs noms, des parties du monde dans lesquelles ils demeurent jusqu'à ce qu'ils puissent être détournés de leur peine et où ils se tiennent pour être convoqués au service [des hommes], et de quels ordres [d'anges] ils tombèrent $\aleph^{18}$. L'on peut raisonnablement se demander si cet ouvrage ne doit pas être identifié avec le fameux Liber qui incipit: Mors anime et desperatio vite, perdu mais mentionné dès le milieu du XII ${ }^{e}$ siècle par la Chronique du Pseudo-Turpin ${ }^{19}$, puis par plusieurs savants des XIII et XIV ${ }^{\mathrm{e}}$ siècles, notamment par l'auteur du Speculum astronomie, qui le considére comme le pire des livres détestables de magie salomonienne ${ }^{20}$. En tout cas, Michel Scot revendique, dans le paragraphe suivant du même chapitre, la composition d'un Liber consecrationis traitant de la manière de conjurer les esprits ${ }^{21}$, qui a de bonnes chances d'être l'un des ancêtres du Liber consecrationum conservé dans différents manuscrits du $\mathrm{xv}^{\mathrm{e}}$ siècle et traduit dans celui de Cambridge sous le titre de Livre des conjuracions. Quant au chiffre de 6666 diables par légion démoniaque, il est cité dans les deux versions du Liber introductorius ${ }^{22}$. Michel Scot énumère par ailleurs une vingtaine de démons qui permettent de supposer que le who's who démonologique dont il dispose est distinct de ceux dont on trouve la trace par la suite : on y reconnaît quelques noms célèbres, communs avec ceux de Wier et du Livre des esperitz (Asmodée, Egym, Belial, Astaroth, Belzebuth, etc.), mais aussi d'autres démons non cités par ces auteurs tardifs, comme Belfegor, Mandrac et Gargifer ${ }^{23}$.

9 2. Dans son Opus tertium composé dans les années 1260, Roger Bacon mentionne, entre autres livres de magie, un Liber de morte anime et un Liber de officiis et potestatibus spirituum $^{24}$.

10 3. Dans son commentaire au De sphera de Jean de Sacrobosco, Cecco d'Ascoli ( $†$ 1327), qui prétend s'inspirer en l'occurrence d'un traité attribué à Zoroastre, parle des 
«quatre esprits de grande vertu qui se tiennent aux points cardinaux [...], nommés Oriens, Amaymon, Paymon et Egim, qui sont des esprits de la hiérarchie majeure et qui ont sous eux 25 légions d'esprits chacun ${ }^{25}$. Ces mêmes rois des démons sont invoqués, lors d'une conjuration visant à provoquer l'amour, dans le livre I, chapitre 6, de la fameuse Clavicula Salomonis, dont la version latine remonte au plus tard au début du XIV siècle mais dont le plus ancien exemplaire conservé est une traduction italienne datée de $1446^{26}$. Et on les retrouve comme destinataires privilégiés des prières des magiciens dans d'autres manuscrits du $\mathrm{xv}^{\mathrm{e}}$ siècle, notamment dans deux passages du Tractatus discipulorum Salomonis super eutuntam et ydeam $^{27}$ et dans un experimentum catoptromantique du ms. d'Oxford dont il a déjà été question plus haut ${ }^{28}$.

11 4. Dans le Liber sacratus attribué à Honorius de Thèbes, un traité de magie théurgique dont je soupçonne qu'il a été écrit, dans la version conservée à l'heure actuelle, dans les années 1330 , en réponse à la répression de la magie exercée par Jean XXII ${ }^{29}$, dans la quatrième partie consacrée aux esprits terrestres, tous mauvais, sont décrits cinq chefs des démons dont la monstruosité est encore plus achevée et complexe que celle de leurs congénères de la Pseudomonarchia, ce qui pourrait indiquer que ces derniers relèvent d'une tradition plus ancienne, sur laquelle le Liber sacratus surenchérit. Ces démons ont des noms qui semblent avoir été majoritairement inventés pour les besoins de la cause: Corniger, roi du Midi, a quatre ministres des quatre points cardinaux nommés Drocornifer, Malifer, Eviraber et Mulcifer. Chacun d'entre eux commande 100 légions de 4500 démons. Mais le prince des ministres de l'Enfer qu'il faut invoquer pour chasser les autres démons des trésors cachés, afin de garder le magot pour soi, est un certain Labadau (identifiable à Abaddon, l'ange destructeur ${ }^{30}$ ?), lequel est assisté d'Asmodée, « qui donne un trésor indestructible dans n'importe quelle monnaie ${ }^{31}$.

5. On conserve, dans quelques rares grimoires $d u \mathrm{xv}^{\mathrm{e}}$ siècle qui ont échappé au bûcher, des listes partielles de démons du type de celle de la Pseudomonarchia. Il y en a sûrement d'autres, mais je n'en ai repéré pour l'instant que deux. Il s'agit de la liste de 11 démons énumérés dans le manuel allemand de «nigromancie » publié par R. Kieckhefer (sigle $M$ dans l'appendice), et d'une liste de 37 démons, malheureusement incomplète du début, que j'ai repérée dans un manuscrit de la Bibliothèque Laurentienne, dont le colophon situe la copie à Rome, en 1494 (sigle F). Cette dernière liste présente l'intérêt majeur de mentionner quelques démons que l'on ne trouve pas ailleurs, mais surtout d'être suivie d'un modus operandi bien mieux adapté que celui de la Pseudomonarchia.

6. Enfin, dans le précieux catalogue de livres de magie que Johannes Trithemius a inséré, en 1508, dans son Antipalus maleficiorum, on trouve deux traités qui relèvent du genre littéraire qui nous occupe : l'un, placé en seconde position du catalogue, juste après la Clavicula Salomonis, et intitulé Liber officiorum, distingue cinq dignités de chefs des démons : des empereurs, des rois, des ducs, des marquis et des comtes ${ }^{32}$; l'autre est décrit de la façon suivante: Et est liber Salomoni adscriptus De officiis spirituum. Magnus et alius ab illo, quem superius nominavi, execrabilis et totus diabolicus, qui sic incipit: «In hoc libro sunt secreta omnium artium $»^{33}$. C'est cette dernière version qui semble la plus proche du Livre des esperitz et du manuscrit publié par Wier sous le titre de Pseudomonarchia doemonum.

Onomastique et hiérarchie démoniaques

14 Ces précédents une fois rappelés, il est temps d'entrer davantage dans l'analyse de nos quatre listes des $\mathrm{Xv}^{\mathrm{e}}$ et $\mathrm{XVI}^{\mathrm{e}}$ siècles, qui donnent les noms, au total, de quelque 107 démons. 
15 L'Enfer, comme le rappelle le début du Livre des esperitz, est une monarchie placée sous l'autorité suprême de Lucifer, l'Étoile du matin considérée à tort par les Pères de l'Église (cf. Isaïe 14, 12-15) puis par les clercs médiévaux, comme un prince des démons ${ }^{34}$.

16 Lucifer est peut-être considéré comme difficilement atteignable par les magiciens du Moyen Âge chrétien. En tout cas, les invocations qui lui sont adressées sont rares et les plus intéressantes d'entre elles sont repérables dans des manuscrits italiens du $\mathrm{xv}^{\mathrm{e}}$ siècle, soit d'une façon indirecte ${ }^{35}$, soit dans le cadre particulier d'un recueil de magie amoureuse d'origine milanaise, où une superbe conjuration a pour destinataire Lucifello, premier des anges, tombé du ciel sous la forme d'un «serpent antique et terrible $»^{36}$. Belzebuth, lui aussi, semble peu invoqué directement, même s'il est appellé deus amoris et représenté comme tel dans la version longue du Liber introductorius de Michel Scot ${ }^{37}$. Satan, malgré son aspect effrayant et son caractère redoutable, apparaît plus fréquemment comme destinataire potentiel des prières de l'officiant dans la magie rituelle: on le trouve mentionné comme tel dans le Tractatus discipulorum Salomonis super eutuntam et ydeam ${ }^{38}$, mais aussi dans le manuel édité par R. Kickhefer, où une longue conjuration de Satan/Mirage vise à l'obtention d'un esprit familier, susceptible de satisfaire les plus chers désirs de l'invocateur ${ }^{39}$.

17 Au total, une vingtaine de nos démons sont des interlocuteurs privilégiés des magiciens. On pense en particulier aux rois des points cardinaux ${ }^{40}$, mais aussi à Astaroth, Belial et Berith ${ }^{41}$, à Byleth ${ }^{42}$ et à Flavos/Flauros, peut-être identifiable à Floron ${ }^{43}$.

18 Je n'insisterai guère sur les noms des autres démons et sur leur possible origine ${ }^{44}$. Je me contenterai seulement de signaler quelques noms signifiants, en rapport avec l'apparence ou les fonctions des démons concernés : Bifrons dans W ; Cerbere dans $C$ et $\mathrm{W}$; Triplex sive Complex, un ange déchu de l'ordre des Principautés devenu magnus dux et marchio dans F, qui apparet in similitudinem angeli pulchri et [...] per eum possunt omnia tripliciter ligari ${ }^{45}$.

19 Dans le Livre des esperitz, la hiérarchie des démons n'est pas très claire, dans la mesure où leur titre n'est pas un critère déterminant de différenciation du volume des troupes qui leurs sont confiées : les rois ont de 100 à 6 légions, 31 en moyenne; les ducs de 80 à 15 légions, 35 en moyenne ; les princes de 36 à 20 légions, 30 en moyenne ; les marquis de 50 à 19 légions, 31 en moyenne ; le "grant seigneur » en a 30 et les deux comtes 25. De ce point de vue, la hiérarchie militaire de la Pseudomonarchia est plus affirmée : les 14 rois ont en moyenne 50 légions, les 6 princes en moyenne 41 légions, les 13 chefs/ présidents (proeses) en moyenne 39 légions, les 23 ducs en moyenne 32 légions, les 14 marquis en moyenne 29 légions, les 12 comtes en moyenne 27 légions, et le chevalier (miles) en a 20. On remarquera que la hiérarchie des titres des démons de la Pseudomonarchia a en partie pour référence l'empire carolingien (le titre de marchio, chef d'une marche frontière, est une innovation de Charlemagne), et que l'absence de tout encadrement intermédiaire dans les armées infernales caractérise nos textes démonologiques, pourvoyeurs de titres ronflants mais peu préoccupés par des problèmes de stratégie ${ }^{46}$.

20 Le Livre des esperitz est très elliptique sur l'apparence physique des démons, apparence qui est au contraire soigneusement décrite dans la Pseudomonarchia. Sur 59 démons ainsi décrits dans le traité édité par Wier, 36 , soit près des deux-tiers, sont des monstres qui combinent à des degrés divers les espèces humaine, animale et angélique: un 
exemple typique de combinaison à cet égard est représenté par Volac, qui a l'apparence d'un garçon avec des ailes d'ange, chevauchant un dragon à deux têtes. Nombreux aussi sont, à l'instar de Satan, les démons à trois têtes animales et/ou humaines: Aym, pourvu de trois têtes de serpent, d'homme et de chat, chevauche une vipère géante qui crache le feu et détruit camps militaires et cités. Significatifs également sont les deux démons, Bélial et Pucel, qui apparaissent comme des anges, afin de donner au magicien un alibi lui permettant de faire croire qu'il a pu se tromper d'interlocuteur ${ }^{47}$ : le Diable, comme chacun sait, est le pater mendacii, le père du mensonge et de l'illusion.

Démons et magie

21 L'illusionnisme est l'une des fonctions essentielles de la magie en général et de nos who's who démonologiques en particulier. Rappelons que les recettes de caractère illusionniste (obtenir un banquet, un château, un bateau, un cheval, un trône volant, ressusciter un mort, être invisible, etc.) représentent le tiers des experimenta du manuel de "nigromancie " publié par R. Kickhefer, les recettes divinatoires $43 \%$ et les recettes psychologiques $19 \%^{48}$. Dans le Livre des esperitz, sur 87 fonctions repérées, ce sont les finalités divinatoires ( $23 \%$ ) qui l'emportent de justesse, mais l'illusionnisme et les techniques imaginaires arrivent en seconde position avec $22 \%$ des fonctions, devant les finalités cognitives (17\%), la recherche du pouvoir (16\%), de la richesse $(7 \%)$, de la santé (7\%), de l'amour et d'autres vecteurs psychologiques (8\%).

Mais c'est surtout la Pseudomonarchia qui est riche d'enseignements sur les fonctions et les motivations de la magie. Là encore, ce n'est pas la recherche du pouvoir qui l'emporte : sur 139 fonctions repérées, la puissance politique, la faveur des grands et la réussite à la guerre n'interviennent que 17 fois, soit dans $12 \%$ des cas. De même, faut-il le rappeler, la magie, même démoniaque, ne sert pas essentiellement à tuer les gens : les démons qui provoquent la mort ne sont invoqués qu'en quatre occasions. Elle ne sert pas non plus à les rendre malade ou à les soigner (deux occurrences seulement), ni à provoquer l'amour, l'amitié ou la haine (15 occurrences, soit $11 \%$ ). En revanche, ce qui domine, et d'une façon assez massive, c'est la recherche du savoir ( $47 \%$ des fonctions) : non seulement le savoir divinatoire qui donne la connaissance du passé, du présent, de l'avenir (26 occurrences, soit $19 \%$ des fonctions) et des choses cachées (à commencer par les trésors cachés: neuf occurrences), et le savoir merveilleux qui permet de transformer l'équilibre des forces de la nature (transmutation des métaux, transformation de l'eau en vin ou le contraire), mais aussi et surtout le savoir et le savoir-faire des hommes ordinaires (39 occurrences, soit $28 \%$ des fonctions) : la connaissance des arts libéraux du trivium et du quadrivium, de la philosophie (voire de la théologie : trois occurrences) et des arts mécaniques. La magie sert donc, avant tout, à avoir des capacités intellectuelles hors du commun, mais en maitrisant d'abord les disciplines enseignées dans le cadre scolaire et universitaire. Dans ce cadre, les capacités divinatoires (19\%) et illusionnistes ( $16 \%$ : cinq occurrences d'invisibilité, six de transport aérien, y compris, dans un cas, de l'âme au Purgatoire) donnent un surcroît de satisfaction, mais ne sont pas indispensables par rapport à ce qui semble l'essentiel : une maîtrise extraordinaire des connaissances ordinaires.

On remarquera simplement, pour finir, la relative cohérence thématique de certaines fonctions des démons, qui confinent même à la tautologie : la magie sert à devenir et à rester un bon magicien. $9 \%$ des fonctions repérées dans la Pseudomonarchia relèvent de cette capacité d'entretien et d'amélioration de l'art magique : neuf démons servent à fournir les meilleurs esprits familiers, deux autres sont utilisés dans les exorcismes et 
contre des esprits encore plus mauvais qu'eux. Enfin, les fonctions de deux démons montrent, s'il en était besoin, que la «nigromancie » de la fin du Moyen Âge et de la Renaissance, même si elle s'en éloigne, ne renie pas totalement la nécromancie de ses origines $^{49}$ : Bune et Bifrons ont respectivement pour fonction d'être capables de faire changer les morts de place, de réunir les démons sur leur tombe (Bune), et d'allumer des chandelles sur lesdites tombes (Bifrons). Ces chandelles, omniprésentes dans les rituels magiques où les indices tendant à prouver l'existence d'« un système de la lumière ", pour reprendre une expression employée par Jacques Chiffoleau ${ }^{50}$, sont assez nettement perceptibles, nous ramènent à notre point de départ, c'est-à-dire à Lucifer.

À Lucifer, en majesté : est-il besoin de dire que dans le genre littéraire qui nous occupe, la majesté est omniprésente, presque trop. On croit rêver, lorsqu'on lit de tels textes, dans la mesure où l'on a bien l'impression d'avoir ici la preuve de l'existence, dans l'Occident des derniers siècles du Moyen Âge et de la Renaissance, d'un véritable défi de la littérature magique à l'égard des autorités de l'Église et de l'État. Même s'ils l'ont fait sous le manteau, les magiciens qui ont diffusé ces textes ont donné à leurs adversaires bien des torches pour se faire brûler. On remarquera cependant que cette majesté démoniaque, concurrente de celle des pouvoirs en place, n'est pas essentiellement une majesté maléfique : même s'ils sont des monstres plus ou moins effrayants, les diables sortent ici de l'Enfer pour assouvir les désirs des hommes, plus que pour répandre le mal sur la terre. Chose curieuse, et même scandaleuse aux yeux des gardiens de l'orthodoxie chrétienne, ces démons semblent plutôt bienveillants, voire bienfaisants. On pense à l'article 23 des condamnations parisiennes de 1398, que Wier reproduit d'ailleurs dans sa somme: "que parmi les démons, il y en a certains qui sont bons, d'autres bénins, d'autres omniscients, et d'autres ni sauvés ni damnés. Erreur $»^{51}$. La survivance effective, chez les magiciens lettrés européens du XIII ${ }^{\mathrm{e}}$ au XVI ${ }^{\mathrm{e}}$ siècle, de cette très ancienne conception démonologique, également attestée à Byzance ${ }^{52}$, ne fait aucun doute lorsqu'on consulte les quelques manuscrits subsistants qu'ils ont possédés. Mais l'on peut se demander s'ils n'adhéraient pas à cette conception d'une façon purement instrumentale, et qui, dans le reste de la société, la partageait avec eux.

Annexeles démons dans quatre listes des $\mathrm{Xv}^{\mathrm{e}}$ et $\mathrm{XVI}^{\mathrm{e}}$ siècles

\section{Sigles}

M : ms. Munich, Clm 849, fos $65 v^{\circ}-65 r^{\circ}$ bis (milieu du $x v^{e}$ s.), éd. Richard Kieckhefer, Forbidden Rites. A Necromancer's Manual of the Fifteenth Century, Stroud, Sutton Publishing, 1997, p. 291-293.

F : ms. Florence, Bibl. Laurent., Plut. 89 sup. 38 , fos $459-467 \mathrm{v}^{\circ}$ (daté de 1494 ).

C : Livre des esperitz, ms. Cambridge, Trinity College, ms. 0.8.29, fos $179-182 v^{\circ}$ (milieu du $\mathrm{XVI}^{\mathrm{e}} \mathrm{s}$.).

W : Johann Wier, Pseudomonarchia dcemonum, Bâle, 1577, col. 911-934.

Acar, magnus comes, 20 lég. F33.

Agaret, duc, 36 lég. C8. Agares, dux primus sub potestate Orientis, 31 lég. W2.

Alocer, dux magnus, 36 lég. W64.

Alphas. Cf. Malphas.

Alugor, magnus dux, M5. Abugor, duc, 27 lég. C18. Eligor, al. Abigor, dux magnus, 60 lég. W12.

Amduscias, dux magnus et fortis, 29 lég. W53.

Amon, marquis, 40 lég. C11. Amon vel Aamon, marchio magnus, 40 lég. W5.

Amoymon, roi du midi, C6. -, rex Orientalis, W. 
Amy, proeses magnus, 36 lég. W61.

Andras vel Vandras, magnus marchio, 30 lég. F20 et W54.

Andrialfis (Vuduch vel -), proeses, 30 lég. F19. Andralphus, marquis C46. Androalphus, marchio magnus, 30 lég. W55.

Ara, dux et marchio, 25 lég. F32.

Arabas vel Accabas vel Irabas, magnus princeps, 20 lég. F24. Orobas, magnus princeps, 20 lég. W58.

Artis, duc, 36 lég. Botis, al. Otis, magnus proeses et comes, 60 lég. W9.

Asmoday, roi, 12 lég. C25. Sydonay, al. Asmoday, rex magnus, 72 lég. W35.

Astaroth, dux magnus et fortis, 40 lég. F7 et W28.

Aveche, rex et dux, 30 lég. F1.

Azo. Cf. Otius.

Bachimy vel Albermi vel Cabeym, dux magnus et fortis, 26 lég. F23. Aym vel Haborym, dux magnus et fortis, 26 lég. W57.

Balam, $c f$. Vaal.

Balpala, dux magnus et fortis, 30 lég. F25. Vapula, dux magnus, 36 lég. W59.

Barbarus, magnus comes et dux, 36 lég. M1. Barbas, prince, 36 lég. C12. Barbatos, magnus comes et dux, 30 lég. W6.

Barthas, prince, 36 lég. C9. Marbas, al. Barbas, proeses, 36 lég. W3.

Bathym. Cf. Machin.

Beal, rex, 6 lég. C7. Bael, rex, 66 lég. W1.

Beduch vel Bamone, magnus marchio, 20 lég. F5.

Belial, rex, 30 lég. F28. - second de Lucifer, 80 lég. W23.

Berich, rex et dux magnus, 26 lég. F7. Bertheth, duc, 26 lég. C36. Berith, dux magnus et terribilis, 26 lég. W27.

Bifrons, 26 lég. W46.

Bille, dux fortis, 26 lég. F3.

Bitur, grand marquis, 36 lég. C28. Sytry, al. Bitru, magnus princeps, 60 lég. W21.

Boab, magnus prceses, 40 lég. F2.

Bonoree, magnus marchio et dux, 19 lég. F6. Roneve, marchio et comes, 19 lég. W26.

Bucal, duc, 28 lég. C46. Pucel, dux magnus et fortis, 48 lég. W38.

Buer. Cf. Gemer.

Bugan, roi, 34 lég. C30. Haagenti, magnus proses, 33 lég. W67.

Bulfas, prince, 36 lég. C10. Pruflas, al. Bufas, princeps et dux, 26 lég. W4.

Bune, grand marquis, 36 lég. C27. - dux magnus et fortis, 30 lég. W24.

Byleth, rex magnus et terribilis, 85 lég. W20.

Caap, prince, 20 lég. C26. Gaap, al. Tap, prceses magnus et princeps, 66 lég. W36.

Cambea, magnus comes, 20 lég. F17. Decarabia vel Carabia, magnus rex et comes, 30 lég. W52.

Carmola, grand prince, 26 lég. C21.

Cason, magnus dux et fortis, 45 lég. M2. Gazon, duc, 40 lég. C14. Gusoyn, dux magnus et fortis, 45 lég. W8.

Caym, magnus proeses, 30 lég. W41.

Cerbere, marquis, 19 lég. C20. Naberus, al. Cerberus, marchio, 19 lég. W17.

Chax, al. Scox, dux et marchio magnus, 30 lég. W37.

Cimeries, magnus marchio, 20 lég. W60.

Coap, prince, 27 lég. C23.

Curson, magnus rex et fortis, 22 lég. M4. Diusion, roi, 24 lég. C17. Pursan, al. Curson, 
magnus rex, 22 lég. W11.

Dam, comte, 25 lég. C37.

Distolas, marquis, 20 lég. C35. Stolas, magnus princeps, 26 lég. W69.

Drap, duc, 80 lég. C24.

Ducay, grand prince, 26 lég. C45.

Egym, rex magnus et fortis, 1000 lég. F29. Equi, C.

Fameis vel Fronone, marchio magnus, 20 lég. F4. Forneus, magnus marchio, 29 lég. W25.

Fenix, marquis, 25 lég. C34. Phoenix, magnus marchio, 20 lég. W68.

Flavos, duc, 20 lég. C32. Flauros, dux fortis, 20 lég. W62.

Focalur, dux magnus, 30 lég. W44.

Forcas, grand prince, 30 lég. C39. Furcas, miles, 20 lég. W39.

Forchas vel Fortas vel Sartii, prceses magnus, 29 lég. F9. Forras vel Forcas, magnus prceses, 29 lég. W29.

Furfur, magnus comes, 25 lég. F10. - grand comte C38. - comes magnus, 26 lég. W30.

Gaeneron, dux fortis, 27 lég. M8. Gomeris vel Caym, dux fortis, 21 lég. F16. Gomory, dux fortis, 26 lég. W51.

Gamygyn, magnus marchio. W47.

Gay-Bezlebuth, C2.

Gemer, roi, 40 lég. C13. Buer, proeses magnus, 50 lég. W7.

Glasyalabolas, al. Caacrinolas vel Caassimolar, magnus proeses, 36 lég. W18.

Goap, rex et princeps Occidentalis W.

Gorsor vel Gorson, dux fortis, 12 lég. F13. Gorsay, duc, 15 lég. C41. Gorson, rex Meridionalis W.

Halphas, comes magnus, 26 lég. W43.

Hanni, prceses magnus, 30 lég. M10.

Ipes. Cf. Vipos.

Judifligei, ductor fortis, 19 lég. F18.

Lambes, magnus rex, 15 lég. F36.

Lanima vel Primam, magnus comes, 20 lég. F26.

Loray, al. Oray, magnus marchio, 30 lég. W13.

Lucifer, $\mathrm{C} 1$.

Lucubar, duc C29.

Machin, duc, 37 lég. C16. Bathym, al. Marthim, dux magnus et fortis, 30 lég. W10.

[M]alphas vel Malapas, prceses magnus, 20 lég. F12. Malpharas, grand seigneur, C40. Malphas, magnus prceses, 40 lég. W32.

Margoas vel Margodas vel Margutas, 20 lég. F11. Margotias, marquis, C44. Marchocias, magnus marchio, 30 lég. W31.

Morax, al. Foraii, magnus comes et prceses, 36 lég. W15.

Murmur, dux et comes W40.

Orias, marchio magnus, 30 lég. W50.

Orient, C4.

Otius, magnus prceses, 27 lég. M3. Azo vel Oze, magnus prceses, 20 lég. F22. Oze, marquis, 25 lég. C44. Oze, proeses magnus, W56.

Paimon, rex, 40 lég. F27. Poymon, 25 lég. C5. Paymon obedit magis Lucifero quam alii reges, 25 lég. W22.

Paragalla, magnus marchio et comes, 30 lég. F34.

Parcas, prince, 30 lég. C31.

Ponicarpo, dux fortis, 30 lég. F35. 
Ras, magnus prceses, 69 lég. F30.

Rau vel Raym, magnus comes, 30 lég. W42.

Salmatis, marquis, 50 lég. C22. Sabnac, al. Salmac, marchio magnus et fortis, 50 lég. W34.

Samon, roi, 25 lég. C42.

Satan, C3.

Saymon vel Zamon, dux fortis et proeses, 30 lég. F21.

Simias vel Sitmas, magnus marchio, 30 lég. F14.

Sucax, magnus marchio, 23 lég. M11.

Taob, magnus et princeps, 25 lég. M6.

Torcha, magnus marchio et dux fortis, F31.

Triplex sive Complex, magnus dux, 43 lég. F37.

Tudiras Hoho, marquis, 31 lég. C43.

Tuveries, marchio magnus et fortis, 30 lég. M9.

Vaal, roi, 39 lég. C33. Balam, rex magnus et terribilis, 40 lég. W63.

Valefar, al. Malaphar, dux, 10 lég. W14.

Vepar, al. Separ, dux magnus, 29 lég. W33.

Vine, magnus rex et comes $\mathrm{C} 45$.

Vipos, comte, 25 lég. C19. Ipes, al. Ayperos, magnus comes et princeps, 36 lég. W16.

Volach, magnus prceses, 27 lég. M7. - 30 lég. F15. Volac, magnus prceses, 30 lég. W50.

Vual, dux magnus et fortis, 37 lég. W66.

Vuduch. Cf. Andrialfis.

Zaleos, magnus comes, W65.

Zepar, dux, 26 lég. W19.

Zymymar, rex Septentrionalis W.

Pièce justificativeLe plus ancien who's who démonologique en français : le Livre des esperitz

Cambridge, Trinity College, ms. 0.8.29, fos $179-182 v^{o}$ (milieu du XvI $\left.{ }^{\mathrm{e}} \mathrm{s}.\right)$.

Cy commence le Livre des esperitz, lequel fut manifesté au saige Salomon a les contraindre en terre et faire obeïr a la volenté humaine, car avant que la science fut trouvee, monstree ne manifestee et revelee audit Salomon, les esperitz faisoi[e]nt trop de maulx et de pestilences sur terre et destrusoi[e]nt plusieurs biens de ce monde, et a humain lignaige faisoi[e]nt plusieurs persecusions; et pour ce que la misericorde de Dieu dona audit Salomon le benefice de ceste saincte science a contraindre lesdits esperitz et faire obeÿr a humaine creature, adfin que leur malice ne regnast plus sur la terre crestienne.

$A u$ commancement du Livre, seront mis les noms des esperitz qui sont gouverneurs et maistres de touz les aultres esperitz; de la region et office de Lucifer; de l'office de Bezlebut; de Satan; des quatre esperitz qui sont gouverneurs des quatre regions et parties du monde, c'est assavoir de l'esperit qui est appellé Orient, de Poymon, d'Equi ; de Veal, le grant roy; de Agarat, duc ; de Barbas, prince ; de Bulfas, prince ; de Amon, marquis ; de Batal, conte ; de Gemen, roy ; de Gazon, duc ; de Artis, prince ; de Machin, duc; de Dicision, roy; de Abugor, duc; de Vipos, conte; de Cerbere, marquis; de Carmola, prince; de Estor, duc; de Coap, prince; de Deas, duc; de Asmoday, roy; de Bitur, marquis ; de Beal, duc; de Forcas, prince; de Furfur, conte ; de Margotias, marquis ; de Oze, prince ; de Lucay, marquis ; de Pucel, duc ; de Jayn, conte ; de Suralet, duc ; de Zagon, roy ; de Dragon, prince ; de Parcas, prince ; de Gorsin, duc ; de Andralfas, marquis ; de Flanos, duc ; de Brial, roy ; de Fenix, marquis ; de Distolas. /fo $179 \mathrm{v}^{\circ}$ / 
[1] Lucifer fut moult bel et de telle stature, quoy sont les aultres bons angelz, et ne demoura mye aux cieulx par l'espace de une heure, car il se enorguillit en regardant et contemplant sa belle grant beaulté en laquelle il fut fourmé ; et touz ceulz qui furent panssantz mal avecque luy furent gectés en Enfer en confusion. Et faict ledit Lucifer, sellon que dient les docteurs de nigromance, et ledit Lucifer preside en Enfer et touz lesdits esperitz d'Enfer oboÿssans a luy comme souverain d'Enfer.

[2] Gay, grant et maulvais esperit, est appellé Bezlebuth, et fut appellé devant le temps de Salomon Anthaon, et est le plus grant d'Enfer aprés Lucifer, et doibt on savoir qu'il regne aux parties d'orient, et celuy qui l'appelle doibt tenir son visaige vers orient et il aparoistra a luy en belle figure et semblance. Il enseigne toutes sciences et donne or et argent a ceulx qui le contraignent a venir, et donne vroye responce de ce que on luy demande, et revele les secretz d'Enfer si on luy demande, et enseigne veritablement les choses mucees $^{53}$ en terre et en mer, et si magnifeste touz tresors qui sont en terre reposantz, et garde des aultres espritz, et doibt estre appellé par beau temps.

[3] Le tiers esperit est appellé Satan, lequel fut fourmé aprés Lucifer, et converse en l'air pres de nous. Iceluy Sathan appiert en gracieuse semblance et habite en septentrion. Celuy qui l'appelle doibt avoir son visaige vers septentrion. Icelluy apiert et a puissance de defformer touz homes et femmes se on luy commande, et se apparoille a faire touz maulx si luy est commandé.

Des quatre esperitz principaulx seront dits les offices en ceste partie, dont le premier ensuit :

\section{Sequitur de primo}

Le premier est appellé Orient et habite en orient. Il tient le nom de la partie du monde.

Pro secundo:

Le second est appellé Poymon et habite en occident.

Pro tertio :

Le tiers est appellé Amoymon et habite es parties meridionales.

Pro quarto:

Le quart est appellé Equi et habite es parties de septentrion.

[4] Officium primi : /fo $180 /$

L'office du premier qui est appellé Orient, est de respondre verité de ce que on luy demande, et si a puissance de consorter touz esperitz et les enseigner a faire, et si apprant au maistre qui le contrainct toute phisicque ; et a dessoubz luy cent legions d'angelz ou de maulvais esperitz.

\section{[5] Poymon}

Poymon appiert en semblance de femme coronnee, moult resplendissante, et chevaulche ung dramagdonere [sic]. Celuy qui le contrainct doibt avoir son visaige vers occident, et il dict verité de ce que on luy demande et apprant toutes sciences au maistre, et manifeste toutes choses mucees, et donne dignités et grandes seigneuries, et faict venir a mercy touz les malveillantz du maistre plainement ; et est seigne[u]r de Xxv legions.

\section{[6] Aymoymon}

Aymoymon est roy et appiert en semblance de demye home et a longue barbe, et porte a son chieff une coronne tres clere, et ayme que on luy face sacriffice, et donne vroye responce de ce que on luy demande ou concede, et donne souvenance de toutes 
sciences et donne grandes dignités en terre et les conferme, et donne bone maniere de bon sens; et a x legions.

Beal est ung grant roy qui est dessoubz et est subgect a Orient, qui est grant et faict home invisible merveilleusement, et donne sa grace a toutes choses ; et soubz luy a seix legions.

41 [8] Agarat

Agarat est duc et appiert benignement, en semblance de home vieulx, et enseigne touz langaiges et donne seigneuries et grandes dignités en terre; et soubz luy a XxxvI legions.

[9] Barthas

Barthas est ung grant prince qui appert en belle figure. Son office est donner responce de ce que on luy demande, et enseigne les choses mucees, et enseigne auxi et faict aux gens ce que on luy demande, et faict home en quelque figure que le maistre vieult, et enseigne parfaictement astronomye ; et a dessoubz luuy Xxxvi legions.

43 [10] Bulfas $/ \mathrm{f}^{\circ} 180 \mathrm{v}^{\circ} /$

Bulfas est ung grant prince. Son office est de faire discordez et batailles, et quant il est bien contrainct, il rend bone responce de ce que on luy demande; et a dessoubz luy xxxvi legions.

44 [11] Amon

Amon est un grant marquis qui appiert en semblance d'une pucelle. Son office est de dire verité des choses passees et advenir en terre, et toute personne de qui on vieult avoir l'amour, il le faict avoir ; et a soubz luy xL legions.

[12] Barbas

Barbas est ung prince qui monstre a entendre le son des oyseaulx et la voix des chiens, et manifeste toutes choses mucees en terre, et les apporte si on luy commande; et a xxxvi legions.

[13] Gemer

Gemer est un grant roy. Son office est de enseigner la vertu des herbes et toutes sciences, et guerir ceulx qui sont malades quant on luy commende, et aussi faict les gens malades; et dessoubz luy a XL legions.

[14] Gazon

Gazon est ung gant duc qui donne vroye responce des choses passes et advenir et des choses presentes, et donne grace et amour envers toutes personnes sur terre, et faict monter en grantz honneurs et dignités ; et a XL legions.

48 [15] Artis

Artis est un grant duc et [a] deux coronnes et une espee en la main. Son office est de respondre de toutes choses que on luy vieult demander et enseigner les choses mucees, et donne bone amour et grace envers toutes persones; et a XXXvi legions.

[16] Machin

Machin est ung grant duc qui est en similitude et semblance d'un home fort, et enseigne la vertu des herbes et des pierres precieuses, et porte le maistre de region en region partout ou le maistre vieult ; et a XxxvII legions.

[17] Diusion

Diusion est ung grant roy qui appiert en semblance d'un bel home, et donne vroye 
responce /fo $181 /$ de ce que on luy demande, et si va querir les tresors mucés en terre quant on luy commande ; et a XxiII legions.

51 [18] Abugor

Abugor est ung grant duc qui appert en semblance d'un beau chevalier, et donne vroye responce de ce que on luy demande et des choses mucees en terre, et donne bone grace envers roys et aultres seigneurs; et a XXVII legions.

[19] Vipos

Vipos est un grant conte qui appert en semblance d'un ange, et faict home saige et hardy, et dit verité de ce que on luy demande ; et a xxv legions.

[20] Cerbere

Cerbere est un grant marquis qui donne parfaict entendement en toutes sciences, et faict home moult grant en honeurs et richesses; et a XIX legions.

[21] Carmola

Carmola est un grant prince qui donne entendement des oyseaulx et a attraper les larrons et meurdriers quant on luy commande. Il faict gens invisibles et dict verité de ce que on luy demande; et a Xxvi legions.

[22] Salmatis

Salmatis est ung grant marquis qui appert en semblance d'un chevalier armé, et faict home en quelque semblance qu'il vieult, et si ediffie forteresses, ediffices, chasteaulx et villes quant on luy commande; et faict apparoir grandes playes en quelque personne ; et a $\mathrm{L}$ legions.

56 [23] Coap

Coap est ung grant prince qui faict avoir femmes et les faict venir la ou l'on vieult et les faict horchaingnes ${ }^{54}$ si on luy commande ; et [a] Xxvir legions.

57 [24] Drap

Drap est ung grant duc qui parle bassement et bletzce [sic] la veue et l'ouye quant on luy commande ; et a IIIIIx legions.

58 [25] Asmoday

Asmoday est un grant roy qui donne ung annel qui [a] si grant vertu qu'il faict celuy qui le porte eureux en toutes choses du monde, et donne vroye responce de ce que on luy demande ; et a soubz luy XII legions.

[26] Caap $/ \mathrm{f}^{\circ} 181 \mathrm{v}^{\circ}$ /

Caap est un grant prince qui appiert en forme d'un chevalier et donne vroye responce de ce que on luy demande, et apporte or et argent de quelque lieu que on luy commande; et a soubz luy xx legions.

60 [27] Bune

Bune est ung grant duc qui faict les corps aler et venir d'un lieu en aultre chemyner, et faict home riche et parler saigement devant toutes gens, et donne vroye responce de ce que on luy demande; et a Xxxv legions.

$61 \quad$ [28] Bitur

Bitur est ung grant marquis qui apert en forme d'ung beau jouvencel et donne l'amor des femmes de quelque lieu que elles soi[e]nt, et destruict villes et chasteaulx si le maistre luy commande, et faict depposer de ses grantz honeurs et dignités de ce monde si le maistre luy commande; et a Xxxvi legions. 
Fenix est un grant marquis qui appert en belle figure, et a la voix moult doulce, et si est courtoys et tres oboïssant a toutes les choses que on luy vieult demander ou commander, et si les faict ignelement et sans dilacion; et a xxv legions.

[35] Distolas

Distolas est ung grant marquis qui appert en belle figure et donne voluntiers responce de ce que on luy demande et commande, et si apporte pierres si on luy commande, et donne au maistre ung cheval qui le porte en une heure cent ou deux ou trois centz lieues ou plus; et a xx legions.

\section{[36] Berteth}

Berteth est ung grant duc qui appiert en belle figure et a une couronne. Il donne vroye responce de ce que on luy demande, et si enseigne a convertir touz manieres de metaux en or ou en argent, et donne seigneuries et conferme si on luy demande; et a XXVI legions.

70 [37] Dam

Dam est ung grant conte qui appert en belle figure, qui apporte or et argent et toutes aultres choses si on luy commande, et faict mourir ou languir toutes personnes que on luy commande. Et si dict touz les secretz des femmes, et si les faict despouiller et dancer toutes nues; et a Xxv legions.

71 [38] Furfur

Furfur est ung grant conte qui appert en guyse d'ung ange et faict avoir l'amor de toutes gens, et faict home saige en astronomie et philosophie.

72 [39] Forcas

Forcas est ung grant prince qui enseigne la vertu des herbes et des pierres precieuses, et 
faict estre invisible et estre saige et bien parlant a toutes gens, et si apporte tresors mucés en terre quant on luy commande ; et a xxx legions.

[40] Malpharas

Malpharas est ung grant seigneur qui ediffie tours et chasteaulx, pontz sur eaues quant on luy commande, et abbatt [sic] et confont soi[e]nt /f $182 \mathrm{v}^{\circ} /$ gens, chasteaulx ou aultres forteresses, et porte d'un lieu en aultre si on luy commande, et oboïst et est courtaix [sic] au maistre qui le contrainct a faire lesdictes choses; et a Xxx legions.

74 [41] Gorsay

Gorsay est ung grant duc qui faict ung home bon oupvrier en ses besoignes et en ses ditz. Il prant larrons et meurdriers et les amaine la ou on luy commende, et faict souffrir paine et tourmant a quelque que l'on vieult ; et a Xv legions.

[42] Samon

Samon est ung grant roy qui appert en semblance d'une belle pucelle. Il donne responce de ce que on luy demande. Il ensaigne les biens et les tresors qui sont mucés et faict avoir l'amour de toutes roynes et femmes parfaictement, soi[e]nt pucelles ou non; et a xxv legions.

76 [43] Tudiras Hoho

Tudiras Hoho est un grant marquis qui appert en semblance d'une belle pucelle et faict home saige en toutes sciences, et si le mue en maniere d'oysel ; et a xxxı legion[s].

77 [44] Oze

Oze est ung grant marcquis qui donne bone responce de ce que on luy demande, et si faict home muer de figure en aultre, et si faict une chose aparoir aultrement qu'il n'est, et si faict une poigne d'estrain ${ }^{55}$ estre ung grant cheval et ung festu estre scainture d'or ou d'argent, et faict les gens forcenés quant on luy commande ; et [a] xxv legions.

[45] Ducay

Ducay est un grant marquis qui apert moult benigenment et donne l'amour des femmes et faict entendre touz langaiges, et porte de lieu en aultre lieu ; et a xxv legions.

[46] Bucal

Bucal est un grant duc qui apert en guyse d'ange et done vroye reponse de ce que on luy demande, et faict aparoir grandes eaues et abysmes en l'air, combien qu'ilz n'y soi[e]nt point ; et a XXVIII legions.

80 Finis. Laudat opus.

Adsit in principio sancta Maria meo.

\section{NOTES}

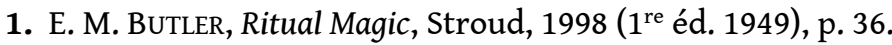

2. R. KIECKHEFER, Forbidden Rites. A Necromancer's Manual of the Fifteenth Century, Stroud, 1997, p. 160-161. 
3. La description du ms. dans le catalogue de M. R. JAMES, The Western Manuscripts in the Library of Trinity College, Cambridge, vol. III, Cambridge, 1902, p. 422-424, est très défectueuse. Notre texte a été copié sur la première partie du sixième ensemble codicologique du volume, qui correspond à un quaternion (fos $\left.179-186 \mathrm{v}^{\circ}\right) \mathrm{de}$

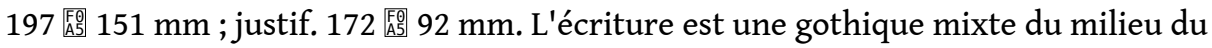
$\mathrm{XVI}^{\mathrm{e}}$ s. Les passages en italiques dans mon édition sont transcrits sur le ms. en lettres oranges et rouges, de plus grand module.

4. R. KIECKHEFER, Forbidden Rites, op. cit., p. 8-10 et 256-276.

5. Sur ce ms., voir F. KLAASSEN, « English Manuscripts of Magic, 1300-1500 : a preliminary Survey ", dans C. FANGER éd., Conjuring Spirits. Texts and Traditions of Medieval Ritual Magic, Stroud, 1998, p. 3-31 (p. 21-24).

6. Éd. Bâle, 1577, col. 910-934. Ce texte est réédité dans l'éd. de Bâle, 1583, et dans l'éd. d'Amsterdam, 1660, des Opera omnia de Wier. Celui de l'éd. d'Amsterdam est disponible sur le site web www.avesta.org/solomon/weyer.htm, avec une brève introduction de J. H. Peterson. Sur Wier et son œuvre, voir J. J. Cobben, Jan Wier, Devils, Witches and Magic (éd. originale publiée en néerlandais en 1960), Philadelphie, 1976, et la traduction anglaise de John Shea : J. Weyer, Witches, devils, and doctors in the Renaissance, éd. G. Mora, Tempe (Ariz.), 1998. Je n'ai pu consulter la thèse récente de M. Valente, Incantamentorum labyrinthis. Un crocevia delle discussioni sulla tolleranza nell'Europa del Cinquecento : Johann Wier e il De prostigiis domonum, Università « La Sapienza », Rome.

7. Wier, Pseudomonarchia, op. cit., col. 911-912.

8. C'est-à-dire Salomon le Magicien ? Emptor, depuis la Réforme grégorienne, désigne en effet l'acheteur simoniaque, ce qui indiquerait une identification possible entre Simon le Magicien et Salomon. Mais Benoît Grévin me suggère que Liber dictus Empto. Salomonis pourrait être une déformation de Liber Emphoras Salomonis, titre qui désignerait le Schem-hamephorash, le nom imprononçable de Dieu de la magie kabbalistique, formé de 72 noms d'anges. Cette autre interprétation est corroborée par le détournement avéré, à la fin du Moyen Âge et à l'époque moderne, du nom divin dans la magie chrétienne, tel que l'on peut l'observer par exemple dans la Goetia, première partie du Lemegeton vel Clavicula Salomonis regis, où un catalogue de 72 démons (les 69 démons de Wier plus trois autres) est réuni sous le titre de Shemamphorash : voir The Goetia. The Lesser Key of Solomon the King. Lemegeton, Book I, Clavicula Salomonis regis, trad. angl. de S. L. Mac Gregor Mathers, $2^{\text {e }}$ éd., Austin, 1997, p. 27-69 (édition charlatanesque de Samuel Weier et de l'Ordo Templi Orientis, agrémentée d'illustrations démoniaques reproduisant des gravures de Louis Breton tirées de la $6^{\mathrm{e}}$ éd. du Dictionnaire infernal de J. Collin de Plancy, Paris, 1863 [réimpr. Genève, Slatkine, 1993]). 9. R. Sсот, The Discoverie of Witchcraft, B. Nicholson éd., Londres, 1886, p. 314-329. Voir également, aux p. 559-563, l'analyse des écarts entre la traduction de Scot et le texte latin de la Pseudomonarchia, écarts qui semblent trouver en partie leur origine dans l'utilisation, de la part de Scot, d'un ms. copié en 1570 par un certain T. R.

10. Voir, en annexe, l'index des démons que j'ai trouvés dans quatre listes des $\mathrm{XV}^{\mathrm{e}}$ et $\mathrm{XVI}^{\mathrm{e}} \mathrm{s}$.

11. Voir notamment, entre autres incohérences, Wier, Pseudomonarchia, op. cit., col. 931 : «Observa horas in quibus quatuor reges scilicet Amoymon rex Orientalis, Gorson rex Meridionalis, Zymymar rex Septentrionalis, Goap rex \& princeps Occidentalis possunt constringi, a tertia hora usque ad meridiem, a nona hora usque ad vesperas. » Ces démons des points cardinaux n'apparaissent guère en tant que tels dans la liste, 
sauf Goap, si l'on veut bien l'identifier à « Gaap, alias Tap, Præses magnus \& Princeps ", qui « in signo Meridiei [sic] apparet » (ibid., col. 923).

12. Ibid., col. 911-912: « Ne autem curiosulus aliquis, fascino nimis detentus, hoc stultitiæ argumentum temere imitari audeat, voces hinc inde prætermisi studio, ut universa delinquendi occasio præcideretur. »

13. Rappelons que dans sa Hiérarchie céleste, le Ps.-Denys l'Aréopagite distingue, dans un ordre hiérachique décroissant, les Séraphins, les Chérubins, les Trônes, les Dominations, les Vertus, les Puissances, les Principautés, les Archanges et les Anges ( $c f$. l'éd. de G. Heil et M. de Gandillac, Paris, 1970, p. 104-132). Or, d'après la Pseudomonarchia, Paymon appartenait avant sa chute aux Chérubins, aux Dominations ou aux Puissances, quatre autres démons faisaient partie des Trônes, deux des Dominations, deux des Vertus, un des Anges, alors que Barbatos et Murmur avaient un statut hybride, entre les Dominations et les Vertus pour le premier, entre les Trônes et les Anges pour le second. Ce statut hybride est partagé, en outre, par les troupes de six démons, Pruflas, Pursan, Paymon, Belial, Forneus et Amy.

14. Il s'agit de Byleth, Marchosias, Focalor, Amy et Phoenix. Au grand scandale de Wier, cela implique que Byleth et Marchosias ont des espoirs de promotion sociale, puisque l'un appartenait aux Puissances et l'autre aux Dominations...

15. J. Delumeau, La Peur en Occident (XIV -XVIII ${ }^{e}$ s.). Une cité assiégée, Paris, 1978, p. 251, se réfère alors à l'évaluation donnée par Wier dans le De præstigiis dœemonum, où il est question de 1111 légions de 6666 démons, soit 7405926.

16. A. Delatte et Ch. Josserand, "Contribution à l'étude de la démonologie byzantine ", dans Mélanges Bidez, Annuaire de l'Institut de Philologie et d'Histoire orientales, t. II, 1934, p. 207-232 (p. 226), signalent que certains traités de magie byzantins indiquent le nombre des démons : groupés eux aussi en légions, ils sont 60 000, 10969 ou 10090 1/2 [sic].

17. Voir l'éd. de C. C. Mc Cown, The Testament of Solomon, Leipzig, 1922, et la trad. et le commentaire de D. DuLING, « The Testament of Solomon (First to Third Centuries AD), a New Translation and Introduction », dans J. H. CHARLESWORTH éd., The Old Testament Pseudepigraphia, vol. I, Apocalyptic Literature and Testaments, Garden City, 1983, p. 935-987. Belzebuth (Beelzebul) et Asmodée sont les seuls démons du Testament de Salomon que l'on retrouve dans le Livre des esperitz et la Pseudomonarchia, mais Satan appartient lui aussi à la tradition judéo-chrétienne antique. Voir le Dictionary of Deities and Demons in the Bible (DDD), K. van der Toorn, B. Becking et P. van der Horst éd., Leyde et al., 1995, col. 197-200, 293-296 et 1369-1380.

18. Munich, Bayerische Staatsbibliothek, ms. Clm 10268, fos $114 \mathrm{r}^{\circ} \mathrm{a}-\mathrm{r}^{\circ} \mathrm{b}:$ : Ptholomeus tamen notantur 48 ymagines stellarum celestis spere in firmamenti, in quibus dominantur morati spiritus sapientissimi, qui sicut sunt in natura diversi sic habent officia diversa que semper operantur inter se et nituntur in inferioribus exercere, ut patet in Libro angelice artis Salomonis et in Libro Adam, etc., in quibus sunt nomina angelorum seu spirituum dampnatorum magne virtutis, qui sunt libri artis nigromancie, cujus titulus talis est : Incipit Liber perditionis anime et corporis ille, quidem liber tractat ordinate cuncta officia demonum et nomina eorum, partes mundi in quibus morantur ad penam quando possunt removeri, quorum stare in servicio convocantis eos, et de quibus ordinibus ceciderunt. » Sur les deux versions du Liber introductorius, voir G. M. Edwards, « The two redactions of Michael Scot's Liber introductorius ", Traditio, vol. XLI, 1985, p. 329-340. 
19. Historia Karoli Magni et Rotholandi ou Chronique du Pseudo-Turpin, C. Meredith-Jones éd., Paris, 1936, réimpr. 1972, p. 226 : « Nigromantia ex qua oriuntur piromantia et ydromantia, et liber sacratus, immo execratus, in aula regis depictus [non] fuit, quoniam libera ars minime habetur. Sciri enim libere potest, sed operari nisi demoniorum familiaritate nullatenus valet. Et iccirco ars adulterina dicitur. Quod etiam ejus nomine approbatur. Mantia enim grece divinatio dicitur, nigro quasi nigra ; unde nigromantia dicitur nigra divinatio. Piros grece rogus, idros limpha ; unde piromantia ignea divinatio, et idromantia limphitica dicitur. Titulus enim nigromantie hic est : Incipit mors anime. "

20. [Ps.-] A. le Grand, Speculum astronomie, chap. XI, P. ZAmBELLI éd., The Speculum Astronomiæ and its Enigma. Astrology, Theology and Science in Albertus Magnus and his Contemporaries, Dordrecht-Boston-Londres,1992, p. 246 : « Sed qui omnium pessimus invenitur est liber quem scribit Aristoteles ad Alexandrum, qui sic incipit : Dixit Aristoteles Alexandro regi : Si vis percipere, etc. Hic est quem quidam vocant Mortem anime. »Ce passage est repris en 1318 par Thaddée de Parme : voir G. Federici Vescovini, «La classification des mathématiques d'après le prologue de l'Expositio super Theorica planetarum de l'averroïste Thaddée de Parme (Bologne, 1318)», dans Manuels, programmes de cours et techniques d'enseignement dans les universités médiévales. Actes du colloque de Louvain-la-Neuve (9-11 sept. 1993), J. Hamesse éd., Louvain-la-Neuve, 1994, p. 137-182 (p. 179). Pour d'autres mentions, voir L. Thorndike, «Traditional Medieval Tracts concerning engraved astrological Images ", dans Mélanges Auguste Pelzer, Louvain, 1947, p. 217-274 (p. 255).

21. Munich, ms. cité, $\mathrm{f}^{\circ} 114 \mathrm{r}^{\circ} \mathrm{b}$ : « Componi etiam Liber consecrationis ex certis experimentis ac doctrina illius, qui dum est a spiritibus consecratus quam cito aperitur $\mathrm{ab}$ aliquo tam cito $\mathrm{ab}$ aperiente auditur clamor illorum spirituum nomine, quorum liber consecratus est qui una voce dicunt corporibus acceptis elementorum : Quid vis? Quid petis ? Quid precipis ? Precipe quod vis. » Un peu plus loin, Michel Scot recommande même à son lecteur d'attacher par une cordelette à son exemplaire du Liber consecrationis une feuille de papier avec les noms du démon interrogé et ses fonctions, afin de ne pas se tromper de destinataire : « ob hoc autem quelibet cartula ipsius libri debet habere cordelam cum carta scripta, continente nomina demonis et ejus officia ne aperiens erret in spiritibus eligendis.

22. Munich, ms. cité, fo $13 \mathrm{v}^{\circ} \mathrm{b}$ (version longue) ; Paris, BnF, ms. nouv. acq. lat. 1401, fo $30 \mathrm{r}^{\circ} \mathrm{a}$ (ms. de la version courte, daté de 1279).

23. Munich, ms. cité, fos $13 \mathrm{v}^{\circ} \mathrm{b}, 17 \mathrm{r}^{\circ} \mathrm{b}$ et $114 \mathrm{v}^{\circ} \mathrm{a}$; Paris, ms. cité, fos $30 \mathrm{r}^{\circ} \mathrm{a}$ et $35 \mathrm{v}^{\circ} \mathrm{a}$.

24. Part of the Opus Tertium of Roger Bacon, A. G. LiTTLE éd., Aberdeen, 1912, p. 48.

25. L. THORNDIKE, The Sphere of Sacrobosco and its Commentators, Chicago, 1949, p. 404 : "Juxta quod debetis intelligere quod isti quatuor spiritus magne virtutis qui stant in cruciatis locis, scilicet in oriente, occidente, meridie et septentrione, quorum nomina sunt ista : Oriens, Amaymon, Paymon et Egim, qui spiritus sunt de majori hierarchia et habent unusquisque sub se 25 legiones spiritum. » Ces démons-rois des points cardinaux se rencontrent également dans la démonologie byzantine, mais avec des noms tout à fait différents : voir L. Delatte, Un office byzantin d'exorcisme, Bruxelles, 1957, p. 89-92. Amaymon doit probablement être identifié à Mammon : voir le Dictionary of Deities and Demons in the Bible, op. cit., col. 1012-1014.

26. Paris, BnF, ms. ital. 1524, fo 211 (voir aussi, dans le même ms., les fos $169 \mathrm{v}^{\circ}-170$ ). La Clavicula [Salomonis] fait partie des traités de magie mentionnés dans le Lucidator de Pietro d'Abano, dont la rédaction a été revue par l'auteur en 1310 : voir G. Federici 
Vescovini, Pietro d'Abano, Tratatti di astronomia : Lucidator dubitalium astronomiae, De motu octavae sphaerae e altre opere, Padoue, 1992, p. 117, 1. 19. Je remercie Julien Véronèse d'avoir attiré mon attention sur ce passage.

27. Florence, BN, ms. II.III. 214 , fos $28 \mathrm{v}^{\circ}-29$. Sur cet étonnant traité, attribué à quatre disciples de Salomon (Fortunatus, Eleazar, Macarus et Toz Grecus) et parfois intitulé De quatuor annulis, notamment dans le Speculum astronomie (op. cit., p. 244), voir

L. Thorndike, "Traditional Medieval Tracts », loc. cit., p. 250-251, et D. Pingree, « Learned Magic in the Time of Frederick II », dans Le scienze alla corte di Federico II, Micrologus, II, 1994, p. 39-56 (p. 45).

28. Oxford, Bodl. Libr., ms. Rawlinson D. 252, fo 18 . Le magicien emploie ici simultanément deux instruments pour attirer ces esprits aériens : Oriens, Egyn et Paymon doivent apparaître dans un même vitrum, et Amaymon dans un autre. Sur cet experimentum, voir A. Delatte, La Catoptromancie grecque et ses dérivés, Liège-Paris, 1932, p. 103-104. Rappelons que la catoptromancie est la divination au moyen de miroirs et d'autres surfaces réfléchissantes.

29. Sur ce texte et sa datation, voir en dernier lieu G. Hedegård, Liber iuratus Honorii. A Critical Edition of the Latin Version of the Sworn Book of Honorius, Stockholm, 2002, et J.-P. Boudet, « Magie théurgique, angélologie et vision béatifique dans le Liber sacratus sive juratus attribué à Honorius de Thèbes ", dans Les Anges et la magie au Moyen Âge, Actes de la table ronde de Nanterre (8-9 décembre 2000), Mélanges de l'École française de Rome. Moyen Âge, t. 114, 2002, fasc. 2, p. 851-890.

30. Voir G. DAVIDSON, A Dictionary of Angels, including the fallen angels, New York, 1967, p. 1-2.

31. G. HEDEGÅRD, Liber iuratus Honorii, op. cit., p. 143, et J.-P. BOUDET, « Magie

théurgique », loc. cit. : « Sunt autem 5 : Corniger, rex meridionalis, et habet 4 ministros in 4 mundi partibus, Drocornifer in oriente, Malifer in occidente, Eviraber in meridie, Mulcifer in septentrione. Et quilibet habent legiones centum, et in qualibet sunt demones 4500 , qui omnes istis 4 obediunt et subduntur. Et isti 4 sunt qui possunt omnes alios spiritus a thesauris absconditis fugare, ligare et constringere, et sunt ministri infernales. Princeps eorum est Labadau, ejus coadjutor est Asmodeus qui dat thesaurum indestructibilem cujuslibet monete. "

32. Éd. dans les Paralipomena opusculorum Petri Blesensi et Joannis Trithemii, Mayence, 1605 , I, 3, p. 293 : « Liber quoque officiorum, qui incipit : Multi sapientes tractaverunt. Quam sit vanus, confictus et mendacio plenus, nemo est vel mediocriter doctus, qui non intelligat. Et quis sapiens illum sine risu vel audire, vel legere posset, in quo demones distinguuntur in quatuor : imperatores, reges quoque multos, duces, marchiones et comites. "

33. Ibid., p. 299-300.

34. Voir G. DAVIDSON, A Dictionary of Angels, op. cit., p. 176, et J. B. RuSSELL, Lucifer : the Devil in the Middle Ages, Ihaca, 1984.

35. On trouve, en effet, dans le Tractatus discipulorum Salomonis super eutuntam et ydeam (Florence, BN, ms. II.III. 214, fo $28 \mathrm{v}^{\circ}$ ), le passage suivant : « His diligenter expletis cum prima Luna fuerit, incipiat exorcizator librum scribere, et sic incipit : Incipit Liber dyagargaricam, et in ipsa pagina designetur figura Luciferi cum VII capitibus, et in circuitus ejus hec litere : "Ego sum Lucifer, qui presum omnibus demonibus terrestribus et infernalibus et ubique commorantibus." "

36. Paris, BnF, ms. ital. 1524, fo 114 : «Volendo ch'alchuna di te s'insonnii, fa questa congiuratione : "Ti congiuro, o Lucifello, il quale dal cielo cadesti, serpente antiquo e 
terribile, tu il quale domatina il primo infra gli altri angeli apparevi, il quale ad nui in terra sei caduto, et dicevi nel cuor tuo, o iniquissimo Diavolo : io ponero la sedia mia sopra l'altitudine in gli altari d'aquilone, et sero simile al Dio altissimo. Io ti congiuro dunque per quella sedia donde cadesti, per il circulo il quale in mano hai tenuto, tu il quale hai seminato gli arbori di scientia, di bene e di male, il quale hai portato le cogitatione di tuo caratteria bono e triste, et per le introdutioni con le qual ingannasti Adam et Eva, et li spogliasti dil'imagine di Dio tu terribile e forte. Corre prestamente et va in questa notte ad la tale figlia di T., intrandogli per fenestra o per buso, et tanto gli stii sopra il suo petto fin che la facci inanimata et acciesa nel amor mio, in tal modo che non la possa dormire, ne requiare, ne pensar se non di me solo, fin che non l'adempia il mio disio, et in questa notte la facci cinque fiate corrumpere e spermatizare per amor mio [...]" ». Ce ms., transcrit et étudié par Florence Gal dans le cadre d'un mémoire de DEA de l'Université de Paris X-Nanterre, doit faire l'objet d'une prochaine publication, sous l'égide du Centre de recherches d'histoire sociale et culturelle de l'Occident (xiiie xviiie s.), dirigé par Henri Bresc.

37. Munich, ms. cité, fo $114 \mathrm{v}^{\circ} \mathrm{a}:$ : Belçebut, deus amoris et princeps multorum spirituum ", y est représenté comme un démon ailé et poilu, aux pattes crochues, tirant des deux mains une corde qui lie par le cou un couple d'amoureux. Voir U. BAUER, Der Liber introductorius des Michael Scotus in der Abschrift Clm 10268 der Bayerischen Staatsbibliothek München. Ein illustrierter astronomisch-astrologischer Codex aus Padua, 14. Jahrundert, Munich, 1983, p. $94-95$ et pl. 22.

38. Florence, ms. cité, fo $28 \mathrm{v}^{\circ}$ : « In secunda vero pagina describatur figura Sathane, cujus primum caput est leonis, secundum hominis, tertium capre, cauda ejus serpentina, flammam ex ore prohiciens, et in circuitu hec litere : "Ego sum Sathanas, qui alicui servientes meos presto", ut dent responsa de presentibus, preteritis et futuris precip[u]e factum est, ut sic recto ordine componantur libri, ut superius diximus et officia eorum scribantur in circuitibus ymaginum quemadmodum in Libro secretorum reperitur, et in Libro magico, et notum fit exorcizatori quod cum ipse loquetur cum prodito Sathana, vel cum voluerit quod ante suam veniat presentiam. Non respiciat nisi contra septentrionem, quoniam ibi est habitatio sua, et ita componantur libri ut officia eorum appareant de foris... »

39. R. KIECKHEFER, Forbidden Rites, op. cit., p. 142-144 et 276-286.

40. Sur Oriens, Amaymon, Paymon et Egim, voir supra. Dans le procès dans lequel il fut impliqué, en 1320, Matteo Visconti fut accusé d'avoir fait fabriquer une statuette d'argent, destinée à envoûter Jean XXII, avec sur la poitrine un signe représentant Saturne et le nom d'Amaymon, démon de l'Occident : voir R. Michel, « Le procès de Matteo et de Galeazzo Visconti. L'accusation de sorcellerie et d'hérésie. Dante et l'affaire de l'envoûtement (1320) ", École française de Rome. Mélanges d'archéologie et d'histoire, t. XX, 1909, p. 269-327 (p. 278).

41. R. Kieckhefer, Forbidden Rites, op. cit., p. 59-60, 81, 83, 87, 100, 111, 117-118, 138-139, $155-156,161,172-174,225-226$ et 358-359. Sur ces démons, voir G. Davidson, A Dictionary of Angels, op. cit., p. 59, 68 et 73 .

42. R. Kieckhefer, ibid., p. 104, 106, 242-243 et 365, assimile Byleth à Lilith, alors qu'il s'agit, me semble-t-il, de deux démons différents (voir G. Davidson, A Dictionary of Angels, op. cit., p. 73 et 174-175). Il y a même un Liber Bileth, conservé dans le ms. Florence, BN, II.III. 214, fos $79 \mathrm{v}^{\circ}-84$, dans lequel, au fo $80 \mathrm{v}^{\circ}$, Bileth est qualifié de rex, au même titre que d'autres démons que l'on retrouve dans nos who's who, notamment Machin/Bathym. 
43. Sur Flavos/Flauros/Hauras, voir G. Davidson, ibid., p. 137. Sur Floron qui figure, à l'instar de Byleth, en bonne place parmi les démons invoqués dans les experimenta de divination par le miroir, voir A. Delatte, La Catoptromancie grecque, op. cit., p. 44-47, et R. Kieckhefer, Forbidden Rites, op. cit., p. 28, 104, 106, 178, 236-239 et 363.

44. Voir B. Grévin, «L'ange en décomposition(s). Formation et évolution de l'onomastique angélique des origines au Moyen Âge ", dans Les Anges et la magie au Moyen Âge, op. cit.

45. Florence, ms. Laurenz., Plut. 89 sup. $38, f^{\circ} 311 v^{\circ}$.

46. De ce point de vue, nos documents paraissent quelque peu en retrait par rapport à la hiérarchie plus élaborée de certains textes grecs. Voir L. Delatte, Un office byzantin d'exorcisme, op. cit., p. 120-127.

47. Voir à ce sujet la confession de Jean de Bar, en 1398, éd. dans J.-P. Boudet, « Les condamnations de la magie à Paris en 1398 ", Revue Mabillon, n. s. 11, t. 73, 2001, p. 121-157, à la p. 155 : «Item, j'ay conjuré ou consacré une pierre de cristal pour y enclore un dyable que je cuidoye estre bon angel. Et disoit un petit enfant qu'il veoit une figure d'evesque qui signoit les choses que je vouloye consacrer. Je m'en repens, etc., et croy fermement que de ce n'estoit riens se non illusion de dyable et que ce $n$ 'estoit point un bon angel qui apparu[t] pour faire telz maulz, ains respute blaspheme et erreur de ce tenir...»

48. R. KIECKHEFER, Forbidden Rites, op. cit., p. 27-28 et 42-125.

49. Sur la confusion entre necromantia (divination par l'invocation de l'esprit des morts) et nigromantia (divination par l'invocation des démons, magie démoniaque) au Moyen Âge, voir, pour une première approche, D. Harmening, Superstitio. Überlieferungsund theoriegeschichtliche Untersuchungen zur kirchlich-theologischen Aberglaubensliteratur des Mittelalters, Berlin, 1979, p. 204-207.

50. C'est lors de la soutenance d'habilitation de Catherine Vincent sur Un monde enluminé : lumière et luminaires dans l'Occident médiéval, Université de Paris I, 27 novembre 1999, que Jacques Chiffoleau a posé la question de savoir s'il existait ou non « un système de la lumière » dans la société chrétienne du XIII ${ }^{\mathrm{e}} \mathrm{au} \mathrm{Xv} \mathrm{e}$. La question mérite en effet d'être creusée et les rituels magiques pourraient constituer un champ important d'investigation en la matière.

51. J.-P. Boudet, « Les condamnations de la magie à Paris en 1398 », loc. cit., p. 151 : «Vicesimus tertius articulus : quod aliqui demones boni sint, alii benigni, alii omniscientes, alii nec salvati nec damnati. Error."

52. A. Delatte et $\mathrm{Ch}$. Josserand, « Contribution à l'étude de la démonologie byzantine », loc. cit., p. 216.

53. Mucees : cachées.

54. Horchaingnes : féminin de horsains (forains, étrangers) ou hors de sens (folles, forcenées)?

55. Poigne d'estrain : fétu de paille. 


\section{RÉSUMÉS}

Le Livre des esperitz, copié au milieu du XVI ${ }^{\mathrm{e}}$ siècle dans le ms. Cambridge, Trinity College 0.8.29 (et publié ici pour la première fois), et la Pseudomonarchia dæmonum, publiée en 1577 par Johannes Wier, peuvent être considérés comme des who's who démonologiques : des listes de démons, avec leur titre, leur apparence physique, leurs fonctions et le volume de leurs troupes, comptabilisé en légions. L'un et l'autre sont les témoins tardifs d'une tradition textuelle appartenant à la magie rituelle, qui remonte au plus tard au XIII ${ }^{\mathrm{e}}$ siècle. Deux autres listes partielles de démons sont conservées dans des manuscrits de magie $d u x^{e}$ siècle. La majesté démoniaque s'y révèle dans toute sa splendeur mais chose curieuse, et même scandaleuse aux yeux des gardiens de l'orthodoxie chrétienne, ces démons semblent plutôt bienveillants, voire bienfaisants. La démonologie magique est en effet rétive à la bipartition de l'univers angélique, propre au christianisme médiéval.

Demonological who's who of the 16th century and their medieval ancestors. The Livre des esperitz, copied in the middle of the sixteenth century in Ms. Cambridge, Trinity College 0.8.29 (and published here for the first time), and the Pseudomonarchia dcemonum, published by Johannes Wier in 1577, may be considered as demonological who's who, i.e. lists of demons, with their titles, physical apparences, fonctions and number of troops, accounted in legions. Both are late testimonies of a textual tradition which have belonged to ritual magic since the 13th century at least. Two other partial lists of demons appear in 15th century magical manuscripts. Demoniacal majesty appears here in its glory but those demons seem rather benevolent, and even beneficent, which is curious and even scandalous for the keepers of christian orthodoxy. Actually, magical demonology resists to the bipartition of angelical universe, proper to medieval christianism.

\section{INDEX}

Mots-clés : démonologie, démons, Johannes Wier, Livre des esperitz, magie rituelle Keywords : demonology, ritual magic

\section{AUTEUR}

\section{JEAN-PATRICE BOUDET}

Université de Paris X-Nanterre, 200 avenue de la République, 92001 Nanterre Cedex ; 111/113 rue de Reuilly, 75012 Paris 